\title{
Compensation Method for Laser-Induced Thermal Effect Using Passive Optics
}

\author{
JunG-GWOn LeE ${ }^{a}$, YeONG-SIK KIM ${ }^{a}$, \\ JEONGYUN KIM ${ }^{a}$ AND JINHO LEE ${ }^{b, *}$ \\ ${ }^{a}$ Department of Physics, Dankook University, Cheonan 31116, Korea \\ ${ }^{b}$ Research Institute of Natural Science and Department of Physics Education, \\ Gyeongsang National University, Jinju-si 52828, Korea
}

Received: 06.08.2020 \& Accepted: 26.10.2020

Doi: $10.12693 /$ APhysPolA.139.56

*e-mail: lee.phys.edu@gnu.ac.kr

\begin{abstract}
We experimentally characterized the laser-induced thermal effect on fused silica and established a method to remove the thermal load using passive optics. Based on the formula analyzed in [J. Kor. Phys. Soc. 69, 1531 (2016)], we were able to determine the specific length of the compensating material to remove the thermal effect. Remarkably, the thermal compensation was observed when the compensating material was placed before or after the material subject to thermal lensing. Our results demonstrate that one single passive optic can be used to overcome the thermal effect for various laser powers without any additional optics.
\end{abstract}

topics: high-power laser, thermal compensation, thermal lensing

\section{Introduction}

There is a growing need to preserve excellent spatial laser beam profiles in response to the rising demand for high-power laser technologies in industrial applications such as laser machining, patterning and drilling as well as in various research fields, including quantum optics, nonlinear optics, fusion, and interferometry [1-8]. As a specific example, in the emerging research field of gravitational wave detection [9-11] (i.e., laser interferometer gravitational wave observatory (LIGO)), the laser beam quality is critical for the detection of small gravitational wave signals (the required laser $M^{2}$ value is $\approx 1.1$ ). However, when optical elements are introduced in a nonuniform spatial profile of the laser (for example, the Gaussian TEM $_{00}$ mode), the absorbed power from the laser causes a refractive index change by temperature distribution (thermal lensing) and surface deformation of the optical element (physical expansion of the optics).

As the input laser beam always generates a thermal gradient across optical elements (even in the case of a flat top spatial profile of the input laser beam), thermal lensing and physical expansion of the optics eventually occur. This, in fact, initiates unwanted laser modes or thermal aberrations. Several theoretical studies on the thermal effects of lasers are reported [12-22] and many techniques have been proposed to overcome the thermal distortion of a laser beam, such as using compensating materials with temperature derivatives opposite of the refractive index $[9,10,23,24]$, laser heating and electrical heating of optical elements [24, 26, 27], tunable liquid crystals [28] and deformable mirrors [29]. Among these efforts, Mueller et al. [9] used a passive material - FK51 glass from Schott with $\frac{\mathrm{d} n}{\mathrm{~d} T}=-6 \times 10^{-6} \mathrm{~K}^{-1}$ - to compensate for thermal lensing in the terbium gallium garnet (TGG) crystal. The FK51 glass was placed behind the TGG and the thermal compensation was monitored with the use of a probe beam. The experiment showed a reduction in the higher-order spatial mode generation in TGG by a factor of 10 . These results demonstrated that a passive material having a negative temperature derivative of the refractive index could be used to compensate for the thermal effect on optics. However, the physical expansion of the material by laser heating was overlooked. It should be noted that the thermal expansion coefficient TGG $\left(9.4 \times 10^{-6} \mathrm{~K}^{-1}[19]\right)$ is comparable to the temperature derivative of its refractive index $\left(\frac{\mathrm{d} n}{\mathrm{~d} T}=20 \times 10^{-6} \mathrm{~K}^{-1}[19]\right)$ and therefore both contributions should be considered equivalent. In addition to the problem of thermal expansion, Mueller et al. [9] assumed that the diameter of the optics was much larger than the beam radius. The case of a larger incident beam size, which is comparable to the size of the optics, should, however, be calculated in a different way.

We have carefully analyzed the temperature profile induced by a Gaussian laser including the physical expansion of optics [18-20]. We have determined the theoretical existence of a general relation 
to establish the material length for the negative temperature derivative of the refractive index of the compensating material for the given optical and thermal coefficients. Depending on where the compensating material is placed (i.e., before or after the material subject to thermal lensing), we could calculate the theoretical values of the lengths of the compensating material working within a moderate power range.

Based on the previous studies, we introduced two materials with negative temperature derivatives of the refractive index, i.e., sodium chloride $(\mathrm{NaCl})$ and barium fluoride $\left(\mathrm{BaF}_{2}\right)$, to compensate for the thermal beam distortion. The thicknesses of the two materials were determined using the previously proposed formulae in [19]. We have noted that the equation could be universally applied to a broad range of laser powers. More importantly, it is irrelevant whether the compensating material is inserted before or after the material subject to thermal lensing as long as the proper length of the compensating material is chosen. A single compensating optical element provides a powerful method to selfadaptively work with various laser powers without the need for any other external parameter.

\section{Experiment}

A diagram of the experimental setup is shown in Fig. 1a. A $632 \mathrm{~nm}$ continuous wave He:Ne laser was used to investigate the thermal lensing effect on fused silica. For all measurements, the laser power of the He:Ne laser was maintained at $10 \mathrm{~mW}$. We chose fused silica $(25.4 \mathrm{~mm}$ diameter and $1.5 \mathrm{~mm}$ thickness) as the test material for thermal lensing because most practical optical elements are made of this material. However, our results are quite generic and applicable also to other materials.

To generate the thermal effect, the light from a self-designed $\mathrm{Nd}: \mathrm{YVO}_{4}$ laser $(1064 \mathrm{~nm})$ of various laser powers $(1,4,8,12$ and $15 \mathrm{~W})$ was incident on fused silica and the beam radius of the He:Ne laser was measured at every $100 \mathrm{~mm}$ interval using a CCD camera. The lens $L_{6}$ in Fig. 1a was used to focus the $\mathrm{Nd}: \mathrm{YVO}_{4}$ laser and generate $30 \mu \mathrm{m}$ laser beam diameters on fused silica. We placed a dichromatic mirror at $45^{\circ}$ before the CCD to avoid the incidence of the $1064 \mathrm{~nm}$ beam on the CCD. The other dichromatic mirror was immediately used after the $\mathrm{He}: \mathrm{Ne}$ laser to combine the $\mathrm{He}: \mathrm{Ne}$ and $\mathrm{Nd}: \mathrm{YVO}_{4}$ laser beams.

Figure 1b shows the He:Ne laser beam diverging owing to the heating of fused silica by the $\mathrm{Nd}_{\mathrm{YVO}} \mathrm{YV}_{4}$ laser. The origin $(0 \mathrm{~mm})$ on the $x$-axis corresponds to the position of fused silica.

As shown in Table I, fused silica has a positive temperature derivative of the refractive index: the increased laser heating results in greater curvature of the convex lens. Since the beam waist was positioned after the fused silica and no thermal compensation was applied, a higher heating effect and rapid divergence appeared, as seen in Fig. 1b. The solid lines depict the curve fittings using [30]:

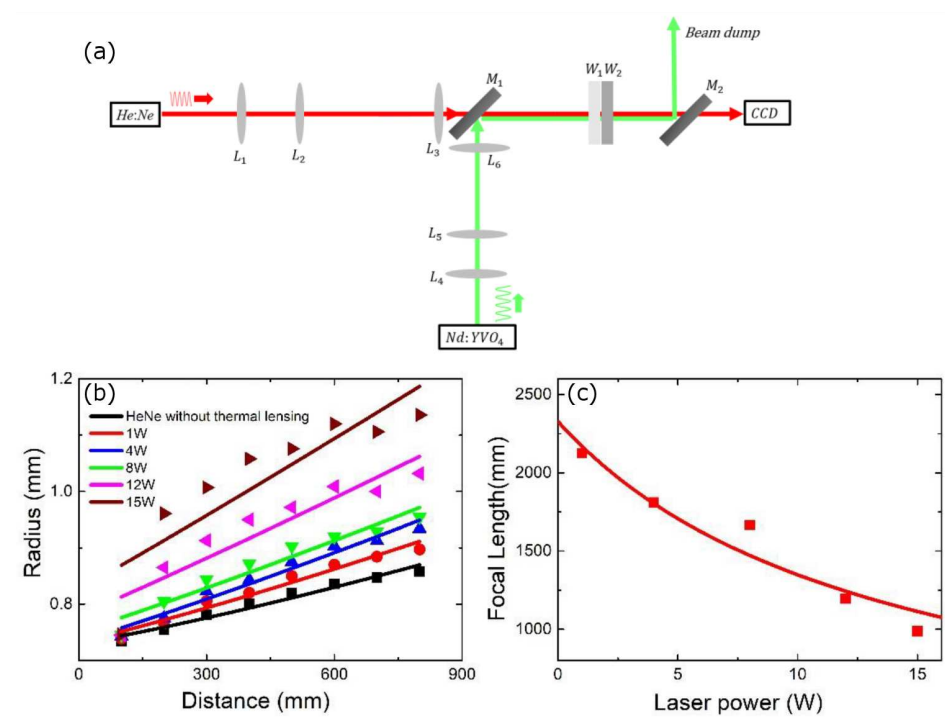

Fig. 1. (a) Schematic of the experimental setup for thermal lens compensation. $W_{1}$ and $W_{2}$ represent fused silica and the compensating material, respectively. We also performed the measurement after swapping the positions of $W_{1}$ and $W_{2}$ (i.e., compensating material is placed first followed by fused silica). $L_{1}\left(L_{4}\right)$ and $L_{2}\left(L_{5}\right)$ in the figure are biconvex lenses to collimate the He:Ne(Nd:YVO 4$)$ laser. The collimated He:Ne and $\mathrm{Nd}_{\mathrm{YVO}_{4}}$ beams are focused on the sample by focusing lenses $L_{3}$ and $L_{6}$ for each of them. $M_{1}$ and $M_{2}$ are dichromatic mirrors transmitting at $632 \mathrm{~nm}$ and reflecting at $1064 \mathrm{~nm}$. (b) Beam waist divergence of the He:Ne laser with and without thermal lensing in fused silica. Scattered plots indicate experimental measurements and the solid lines are fitting curves. (c) Focal length variation of fused silica with increasing the laser power. The solid red line is the curve fitting with $1 /(a+b x)$, where $a$ and $b$ are fitting parameters and $x$ is the laser power. 
Optical and thermal parameters of various optical materials determined in [19].

TABLE I

\begin{tabular}{c|c|c|c|c|c}
\hline \hline Material & $n$ & $\frac{\mathrm{d} n}{\mathrm{~d} T} \times 10^{-6}[1 / \mathrm{K}]$ & $\alpha \times 10^{-2}[1 / \mathrm{m}]$ & $\kappa[\mathrm{W} / \mathrm{mK}]$ & $\alpha_{\mathrm{th}} \times 10^{-6}[1 / \mathrm{K}]$ \\
\hline fused silica & 1.4492 & 10.1 & 0.10 & 1.38 & 0.5 \\
$\mathrm{NaCl}$ & 1.53153 & -40.83 & 0.07 & 1.15 & 44 \\
$\mathrm{BaF}_{2}$ & 1.4685 & -15.2 & 3.21 & 11.72 & 18.1
\end{tabular}

$$
\omega\left(z-z_{0}\right)=\omega_{0} \sqrt{1+\left(\frac{z-z_{0}}{z_{R}}\right)^{2}},
$$

where $\omega(z)$ is the radius of the laser at $z, \omega_{0}$ is the smallest beam radius (beam waist) and $z_{0}$ is the position of the beam waist. The Rayleigh range is defined as $z_{R}=\frac{\pi \omega_{0}^{2}}{\lambda}(\lambda=632 \mathrm{~nm}$ - the wavelength of the He:Ne laser). In these studies, we used $\omega_{0}$ and $z_{0}$ as the fitting parameters.

The ABCD matrix [30] for the simple lens is given as [30]:

$$
\left(\begin{array}{ll}
A & B \\
C & D
\end{array}\right)=\left(\begin{array}{cc}
1 & 0 \\
-\frac{1}{f} & 1
\end{array}\right)
$$

and then the complex composite $q$ is defined using $\omega(z)$ and $R(z)$ (the radius of the beam at position $z)$ as follows [30]:

$$
\frac{1}{q(z)}=\frac{1}{R(z)}-\frac{\mathrm{i} \lambda}{\pi \omega(z)^{2}}
$$

We can relate the value $1 / q_{\text {out }}$ obtained immediately after the lens, with the value $1 / q_{\text {in }}$ obtained immediately before the lens, as [30]:

$$
q_{\text {out }}=\frac{A q_{\text {in }}+B}{C q_{\text {in }}+D} .
$$

Therefore, the focal length of the thermal lens can be calculated for each laser power, as shown in Fig. 1c. The scatter plots (Fig. 1c) are based on (3) and show that the focus becomes shorter with increasing the laser power. Following our previous analysis of thermal lensing (see (16) in [20]), the focal length variation induced by the laser power is proportional to $\frac{1}{a+b x}$, where $a$ and $b$ are constants (fitting parameters) and $x$ is the laser power. As indicated by the solid red line in Fig. 1c, one can see the excellent agreement between the fitted results and those of our experimental measurement.

In [19], we derived the analytical expression for temperature for the cylindrical geometry using the Bessel functions and the optical path length changes along the radial direction, including the physical expansion of the optics, were calculated based on the temperature profile of the optics. If the compensating material has the same radial dimension as the thermal lensing optics, it is easy to find the condition to achieve a zero optical path length $\Delta l(r)+\Delta l^{\prime}(r)=0$, where $\Delta l(r)$ and $\Delta l^{\prime}(r)$ are the optical path lengths through the thermal optics and the compensating material, respectively, given as a function of the radius.
In this way, we can determine the proper length of the compensating material [19]. For this purpose, we can use the expression

$l=-\frac{1}{\alpha^{\prime}} \log \left[1+\frac{\frac{\mathrm{d} n}{\mathrm{~d} T}+\alpha_{\mathrm{th}}(n-1)}{\frac{\mathrm{d} n^{\prime}}{\mathrm{d} T}+\alpha_{\mathrm{th}}^{\prime}\left(n^{\prime}-1\right)} \frac{\kappa^{\prime}}{\kappa} \frac{1-\mathrm{e}^{-\alpha l}}{\mathrm{e}^{-\alpha l}}\right]$

to obtain the length when the compensating material is placed after the thermal optics or, if it is placed before the thermal optics, one uses other expression

$l^{\prime}=-\frac{1}{\alpha^{\prime}} \log \left[1+\frac{\frac{\mathrm{d} n}{\mathrm{~d} T}+\alpha_{\mathrm{th}}(n-1)}{\frac{\mathrm{d} n^{\prime}}{\mathrm{d} T}+\alpha_{t h}^{\prime}\left(n^{\prime}-1\right)} \frac{\kappa^{\prime}\left(\mathrm{e}^{-\alpha l}-1\right)}{\kappa}\right]$

Above, $\mathrm{d} n / \mathrm{d} T$ is the temperature derivative of the refractive index. Next, $\alpha, \alpha_{\mathrm{th}}, n$, and $\kappa$ are the absorption coefficient, the thermal expansion coefficient, the refractive index, and the thermal conductivity of the optics, respectively. The presence of the prime symbols of each parameter and, respectively, their absence indicates the properties of the material subjected to thermal lensing and those of the compensating optics, respectively. The optical path length is given by [19]:

$$
\Delta l(r)=\int_{0}^{L}\left(\frac{\mathrm{d} n}{\mathrm{~d} T}+\alpha_{\mathrm{th}}(n-1)\right) \Delta T(r, z) \mathrm{d} z,
$$

where $\Delta T(r, z)$ and $L$ are the temperature rise at the coordinate $(r, z)$ and the longitudinal length of the optics, respectively. Importantly, the total sum of $\frac{\mathrm{d} n^{\prime}}{\mathrm{d} T}+\alpha_{t h}^{\prime}\left(n^{\prime}-1\right)$ should be negative, not just the $\frac{\mathrm{d} n^{\prime}}{\mathrm{d} T}$ value, to compensate for the thermal load from the test optics. Once the material having a negative value of $\frac{\mathrm{d} n^{\prime}}{\mathrm{d} T}+\alpha_{t h}^{\prime}\left(n^{\prime}-1\right)$ is determined, we can calculate the length $l$ or $l^{\prime}$ of the compensating material which will apply to all higher-order thermal distortions. Additionally, this length will compensate for the beam distortion from the thermal lens for any laser power because (5) and (6) do not include power-dependent terms, as aforementioned in [19].

\section{Results and discussion}

To minimize the thermal effect induced by the $\mathrm{Nd}: \mathrm{YVO}_{4}$ laser heating on fused silica, we chose $\mathrm{NaCl}$ as the compensating material because it has a very large negative temperature derivative of the refractive index $-40.83 \times 10^{-6} \mathrm{~K}^{-1}$, as shown in Table I. Based on the optical and thermal parameters listed in Table I, we calculated the thickness 


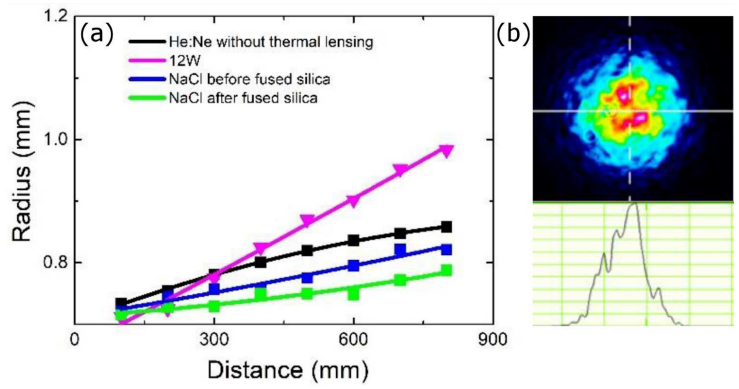

Fig. 2. (a) Thermal compensations (blue and green) using $\mathrm{NaCl}$ for $12 \mathrm{~W} \mathrm{Nd}: \mathrm{YVO}_{4}$ laser heating. (b) Spatial beam profile after compensation.

of $\mathrm{NaCl}$ using (5) and (6). The required thickness of the $\mathrm{NaCl}$ window is $\approx 1.4 \mathrm{~mm}$ for both placements (i.e., before and after fused silica).

We performed the experiment with a commercially ready-made $1.5 \mathrm{~mm} \mathrm{NaCl}$ window. Figure $2 \mathrm{a}$ shows the beam diverging (magenta) from the $12 \mathrm{~W}$ $\mathrm{Nd}: \mathrm{YVO}_{4}$ laser heating. The black curve is the original beam divergence corresponding to the He:Ne laser without thermal lensing. The blue and green curves correspond to compensation with two different configurations of the $\mathrm{NaCl}$ window. We observed excellent thermal compensation for both cases. However, the original Gaussian beam profile of the He:Ne laser was severely distorted after traveling through $\mathrm{NaCl}$, as shown in Fig. 2b. This might be caused by the fact that $\mathrm{NaCl}$ is hygroscopic and therefore very vulnerable to moisture, even though it can withstand temperatures up to $400^{\circ} \mathrm{C}$.
To preserve high beam quality along with thermal compensation, we discarded $\mathrm{NaCl}$ and chose $\mathrm{BaF}_{2}$ as another good candidate. Although it has a smaller negative $\frac{\mathrm{d} n}{\mathrm{~d} T}$ value when compared with $\mathrm{NaCl}$, its thermal expansion coefficient is also smaller, as shown in Table I and thus, a large compensating effect can be expected. As previously mentioned, the negative value of the sum of $\frac{\mathrm{d} n^{\prime}}{\mathrm{d} T}$ and $\alpha_{\mathrm{th}}^{\prime}\left(n^{\prime}-1\right)$ is a critical factor to compensate for positive thermal lensing. The value calculated using (5) and (6) is approximately $0.8 \mathrm{~mm}$ regardless of whether $\mathrm{BaF}_{2}$ is placed before or after fused silica. For the measurement, we used $1 \mathrm{~mm}$-thick $\mathrm{BaF}_{2}$ from Crystran Ltd. Figure $3 \mathrm{a}$ and b represents the thermal compensations for the two different positions of $\mathrm{BaF}_{2}$ and for several laser powers (given by red, blue, green, magenta and brown colors). The black curve in Fig. 3 corresponds to the He:Ne laser without thermal lensing. All the curves in both cases are very close to that of the original He:Ne curve (black), indicating that our scheme, based on the theoretical results of (5) and (6), is well suited to a real situation. Small discrepancies between the compensated He:Ne beams (red, blue, green, magenta and brown) and the original He:Ne beam (black) in Fig. 3a and b could be due to a small mismatch between the optimal thickness of $\mathrm{BaF}_{2}(0.8 \mathrm{~mm})$ and the actual thickness $(1 \mathrm{~mm})$ from the measurement as well as from the beam divergence when traveling through the optics. The derivation of (5) and (6) in [19] had assumed that the periphery of the optics is cooled by a heat sink, i.e., it is maintained at a constant temperature, while the boundary condition
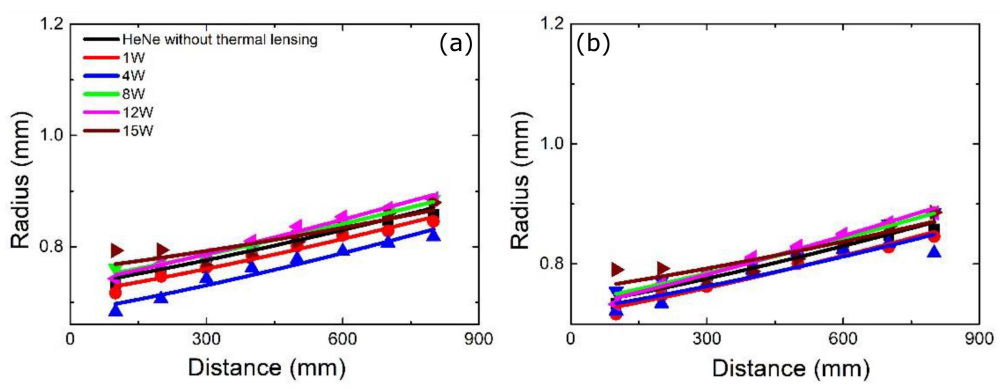

(c)
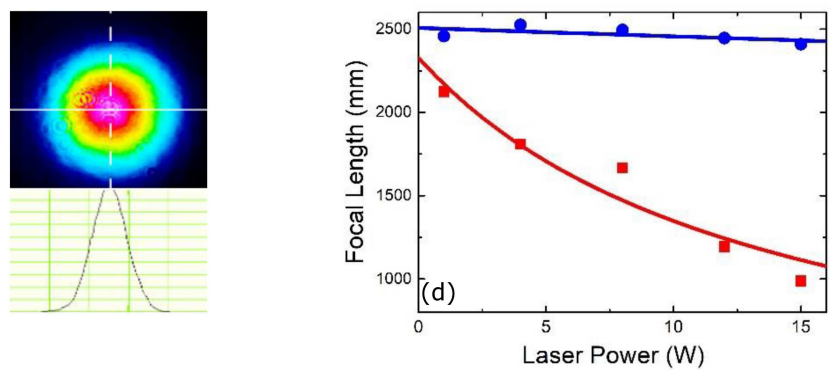

Fig. 3. Thermal compensations using $\mathrm{BaF}_{2}$ placed (a) before and (b) after fused silica for various laser powers. For easy comparison, the $y$-axis scale is the same as that in Fig. 1b. (c) Beam profile of the He:Ne laser after thermal compensation. (d) Focal length variations with (blue) and without (red) thermal compensation when $\mathrm{BaF}_{2}$ was placed after fused silica. (Similar focal length variations were acquired for the different $\mathrm{BaF}_{2}$ positions.). 
of the two sides of the cylindrical geometry of the optics was approximated as $\left.\frac{\mathrm{d} T}{\mathrm{~d} z}\right|_{(\text {at } z=0 \text { and } l)}=0$. However, the air convection, including the StefanBoltzmann radiation, at the two sides of the cylindrical geometry of the optics should be considered to obtain a higher order of precision in the length calculation of the thermal compensating material. Apart from these facts, comparing Fig. $3 a$ and $b$ with Fig. 1b, we can see a good agreement with the theoretical results in both cases. Moreover, the spatial beam profile of the original $\mathrm{TEM}_{00}$ Gaussian He:Ne laser was preserved, as shown in Fig. 3c.

For a more systematic analysis, we calculated the focal length of the system using (3) and for easy comparison, we plotted the thermally compensated focal lengths with the focal lengths under thermal effect alone, as shown in Fig. 3d. In the case of thermal lensing, the focal length changes are inversely proportional to the input laser power, as aforementioned and shown in Fig. $3 \mathrm{~d}$ in red. In the case of good thermal compensation, the focal length would be quite large and remain approximately constant with a change in the laser power because there is no power dependency in thermal compensation, as mentioned and shown in Fig. 3c. Our results demonstrate that thermal lensing can be effectively compensated for a broad range of $\mathrm{Nd}: \mathrm{YVO}_{4}$ laser powers by simply inserting a single optic.

\section{Conclusion}

The use of (5) and (6) (or formula (16) and (18) in [19]) is essential for determining the length of the compensating material. When the proper length of the material is determined, a single passive material can be introduced before or after fused silica to adaptively compensate for thermal lensing without adjusting any external parameters for different laser powers. This is important because if a single compensating optic can remove most of the thermal effects and operate without any external parameters, the system design can be more compact and cheaper. The analysis in this manuscript can be directly applied to the evaluation of a proper compensating material.

It should be noted that our present result focuses on removing thermal lensing from a highpower laser.

\section{Acknowledgments}

This research was supported by the Basic Science Research Program through the National Research Foundation of Korea (NRF) funded by the Ministry of Education (2016R1D1A1B03931391).

\section{References}

[1] J.D. Mansell, J. Hennawi, E.K. Gustafson, M.M. Fejer, R.L. Byer, D. Clubley, S. Yoshida, D.H. Reitze, Appl. Opt. 40, 366 (2001).
[2] T. Imran, M. Hussain, Acta Phys. Pol. A 133, 28 (2018).

[3] J.L. Pura, J. Souto, J. Jimenes, Opt. Lett. 45, 1667 (2020).

[4] K.L. Wlodarczyk, J. Schille, L. Naumann et al., J. Mater. Process. Technol. 285 (2020).

[5] F.Z. Escola, N. Mingolo, O.E. Martines, J.J. Rocca, C.S. Menoni, Appl. Opt. 26, 7233 (2019).

[6] B. Jaeggi, S. Remund, R. Streubel, B. Goekce, S. Barcikowski, B. Neuenschwander, J. Laser Micro. Nanoeng. 12, 267 (2017).

[7] M.N. Nadimi, T. Waritanant, A. Major, Appl. Phys. B 124170 (2018).

[8] L. Andrus, A. Ben-Yakar, Appl. Opt. 59, 8806 (2020).

[9] G. Mueller, R.S. Amin, D. Guagliardo, D. McFeron, R. Lundock, D.H. Reitze, D.B. Tanner, Class. Quant. Gravity 19, 1793 (2002).

[10] M. Adier, F. Aguilar, T. Akutsu et al., Gen. Relativ. Gravity 46, 1749 (2014).

[11] T. Hardwick, V.J. Hamedan, C. Blair, A.C. Green, D. Vander-Hyde, Class. Quant. Gravity 37, 205021 (2020).

[12] W. Koechner, Appl. Opt. 9, 2548 (1970).

[13] U.O. Farrukh, A.M. Buoncristiani, C.E. Byvik, IEEE J. Quantum Electron. 24, 2253 (1988).

[14] M.E. Innocenzi, H.T. Yura, C.L. Fincher, R.A. Fields, Appl. Phys. Lett. 56, 1831 (1990).

[15] A.K. Cousins, IEEE J. Quantum Electron. 28, 1057 (1992).

[16] M. Schmid, T. Graf, H.P. Weber, J. Opt. Soc. Am. B 17, 1398 (2000).

[17] H.S. Carslaw, J.C. Jaeger, Conduction of Heat in Solids, Oxford Univ. Press, Oxford 1948.

[18] J. Lee, D.H. Reitze, Opt. Express 23, 2591 (2015).

[19] G.P. Kwon, J. Lee, J. Kor. Phys. Soc. 69, 1531 (2016).

[20] M. Kim, G.-P. Kwon, J. Lee, Curr. Opt. Photon. 1, 649 (2017).

[21] W. Koechner, Solid-State Laser Engineering, Ch. 7, Springer-Verlag, Berlin 1998.

[22] A. Gharaati, P. Elahi, S. Cari, Acta Phys. Pol. A 112, 891 (2007).

[23] V. Quetschke, J. Gleason, M. Rakhmanov et al., Opt. Lett. 31, 217 (2006).

[24] E. Wyss, M. Roth, T. Graf, H.P. Weber, IEEE J. Quantum Electron. 38, 1620 (2002). 
[25] M.A. Arain, V. Quetschke, J. Gleason et al., Appl. Opt. 46, 2153 (2007).

[26] Z. Liu, P. Fulda, M.A. Arain, L. Williams, G. Mueller, D.B. Tanner, D.H. Reitze, App. Opt. 52, 6452 (2013).

[27] R. Lawrence, D. Ottaway, M. Zucker, P. Fritschel, Opt. Lett. 29, 2635 (2004).
[28] S. Sato, Jpn. J. Appl. Phys. 18, 1679 (1979).

[29] J. Schwarz, M. Geissel, P. Rambo, J. Porter, D. Headley, M. Ramsey, Opt. Express 14, 10957 (2006).

[30] A.E. Siegman, Lasers, University Science Books, Mill Valley (CA) 1986. 